\title{
A Catalog of Orthogonally Blocked Three-Level Second-Order Designs With Run Sizes $\leq 100$
}

\author{
NAM-KY NGUYEN ${ }^{1}$ AND ALOKE DEY ${ }^{2}$ \\ ${ }^{1}$ Vietnam Institute for Advanced Study in Mathematics and VNU International \\ School, Hanoi, Vietnam \\ ${ }^{2}$ Indian Statistical Institute, New Delhi, India
}

\begin{abstract}
Box-Behnken designs form a very popular class of three-level second-order designs when the number of factors is small, typically seven or less. For larger number of factors these designs are not as popular because then these designs require a large number of runs. This article provides a catalog of three-level second-order designs for 5-11 factors with run sizes $\leq 100$. All the designs reported can be orthogonally blocked and are seen to have high $D$-efficiencies.
\end{abstract}

AMS Subject Classification: 62K20.

Keywords: D-efficiency; Response surface designs; Regular graph designs; Resolvable incomplete block designs; Rotatability measure $Q^{*}$.

\section{Introduction}

Box and Behnken (1960) introduced a class of three-level second-order designs, known as Box-Behnken designs, hereafter called BBDs, for fitting a second-order response surface model. BBDs and the central composite designs (CCDs) of Box and Wilson (1951) subject to the appropriate choice of factor levels satisfy several goodness properties judged as essential for a second-order design, such as requiring a minimum number of levels for each of the factors, having a simple data pattern, being rotatable or near-rotatable, being able to be orthogonally blocked, and ensuring simplicity of calculation leading to simplicity in the interpretation of the results (see Box and Draper 2007). As such, BBDs, particularly those with three to seven factors, are very popular second-order designs. BBDs with eight or more factors are less popular as these designs require an excessive number of runs (120-384 runs without center runs for 9-16 factors). Smaller orthogonally blocked three-level secondorder designs have been proposed recently by several authors; see for example, Nguyen and Borkowski (2008) and Dey and Kole (2013), where more references can also be found. The purpose of this article is to provide additional orthogonally blocked three-level designs for 5-11 factors in 100 runs or less with high $D$-efficiency and high $Q^{*}$ rotatability measure (Draper and Pukelsheim 1990).

Received 16 December 2013; accepted 22 July 2014.

Address correspondence to: Dr. Nam-Ky Nguyen, Vietnam Institute for Advanced Study in Mathematics, Hanoi, Vietnam. Email: nknam@ viasm.edu.vn 


\section{Method of Construction}

All designs in this article are constructed from resolvable incomplete block designs (IBDs). Recall that an IBD with parameters $(v, k, r)$ is an arrangement of $v$ treatments in $b(=v r / k)$ blocks, each of size $k(<v)$ such that each treatment occurs in $r$ blocks and each treatment occurs at most once in any block. An IBD is said to be $t$-resolvable if its blocks can be divided into $s$ replicate sets of blocks, each of which is an IBD with parameters $(v, k, t)$, that is, $r=t s$. A 1-resolvable IBD is a resolvable IBD. See Dey (2010) for more on IBDs or Nguyen and Blagoeva (2010) for a brief introduction to this subject. The advantage of using a resolvable IBD in conjunction with the runs of a two-level factorial with levels \pm 1 or, a suitable fraction of a two-level factorial, is that orthogonal blocking can be achieved without any extra effort. Note that since the information matrix of an orthogonally blocked response surface design under a model that includes the block effects, apart from the surface parameters, equals the information matrix under a model with no block effects, the efficiency of an orthogonally blocked design equals the corresponding efficiency of an unblocked design.

Let $\lambda_{i j}(i \neq j)$ be the number of blocks in an IBD in which both treatments $i$ and $j$ concur. If the $\lambda_{i j}$ 's differ by at most 1 , the IBD is called regular graph design (RGD) by John and Mitchell (1977), who conjectured that $D$-, $A$-, and $E$-optimal IBDs are also RGDs. RGDs thus include balanced IBDs (IBDs whose $\lambda_{i j}$ 's are all the same) and all IBDs whose $\lambda_{i j}$ 's differ by 1 . All IBDs used in this article are RGDs.

All BBDs except the one for 11 factors are constructed by superimposing the twolevel factorials with levels \pm 1 onto treatments in each block of an IBD (which can be resolvable or nonresolvable). On the other hand, all designs in this article are constructed by superimposing a two-level fractional factorial and its foldover onto treatments in each block of a resolvable IBD. As an example, suppose we start with the following two-resolvable RGD with parameters $(v, k, r)=(6,3,4)$, whose treatments are labeled $0,1, \ldots, 5$ with the blocks

$$
(2,3,4),(0,5,2),(0,1,3),(1,4,5) ;(0,4,5),(1,3,5),(0,1,2),(2,3,4)
$$

Here, the first four blocks constitute a replicate set and the next four blocks constitute the second replicate set. Each treatment appears twice within a replicate set. If we now impose a half fraction of a $2^{3}$ factorial, say $(1,1,1),(1,-1,-1),(-1,1,-1)$, and $(-1,-1,1)$ onto treatments in each odd block and its foldover in each even block, we get the following six-factor, three-level, second-order design in two orthogonal blocks (without the center points):

\begin{tabular}{cccccc}
0 & 0 & \pm 1 & \pm 1 & \pm 1 & 0 \\
$\mp 1$ & 0 & $\mp 1$ & 0 & 0 & $\mp 1$ \\
\pm 1 & \pm 1 & 0 & \pm 1 & 0 & 0 \\
0 & $\mp 1$ & 0 & 0 & $\mp 1$ & $\mp 1$ \\
\hline \pm 1 & 0 & 0 & 0 & \pm 1 & \pm 1 \\
0 & $\mp 1$ & 0 & $\mp 1$ & 0 & $\mp 1$ \\
\pm 1 & \pm 1 & \pm 1 & 0 & 0 & 0 \\
0 & 0 & $\mp 1$ & $\mp 1$ & $\mp 1$ & 0
\end{tabular}

Here, $( \pm 1 \pm 1 \pm 1)$ represents the four points of a half fraction of a $2^{3},(\mp 1 \mp 1 \mp 1)$ represents the foldover of this half fraction, and 0 represents a column vector of four 0 's. 
It is easy to verify that this orthogonally blocked design has $\Sigma x_{i}^{2} x_{j}^{2}=\frac{1}{2} 2^{3} \lambda_{i j}$ where $\lambda_{i j}$ is the concurrencies of the mentioned RGD.

With reference to an $n$-run second-order design $d$ involving $m$ factors, let $X_{d}$ denote the $n \times p$ design matrix of $d$, where the $i$ th row of $X_{d}$ is written as

$$
\left(1, x_{i 1}^{2}, x_{i 2}^{2}, \ldots, x_{i m}^{2}, x_{i 1}, x_{i 2}, \ldots, x_{i m}, x_{i 1} x_{i 2}, \ldots, x_{i, m-1} x_{i m}\right)
$$

and $p=(m+2)(m+1) / 2$. Then the $X_{d}^{\prime} X_{d}$ matrices of the designs in this article, the augmented-pair designs (Morris 2000), and small CCDs (Nguyen and Lin 2011) have the form

$$
\left(\begin{array}{l|l}
A & 0 \\
\hline 0 & B
\end{array}\right)
$$

where $A$ is a square matrix of order $m+1$ and $B$ is a square matrix of order $m+\left(\begin{array}{c}m \\ 2\end{array}\right)(=$ $\left.\frac{1}{2} m(m+1)\right)$. The quadratic effects of these designs are always orthogonal to all main and interaction effects. Nguyen and Lin (2011) called this property the orthogonal quadratic effect (OQE) property. Unlike BBDs (except the one for 11 factors) and CCDs, where the matrix $B$ in Eq. (1) is a diagonal matrix, certain $\Sigma x_{i} x_{j} x_{l}$ terms in the off-diagonal elements of $B$ will be nonzero.

To construct the designs in this article we first construct a large number of resolvable RGDs using the algorithm of Nguyen (1994) (see http://designcomputing.net/gendex/ibd/ and http://iasri.res.in/design/ibd/ibd.htm). For each RGD we construct an orthogonally blocked three-level second-order design using the method in the previous paragraphs and compute its $D$-efficiency relative to a $D$-optimal continuous (approximate) design measure over an $m$-ball of radius unity, with $m$ being the number of factors in the design. The design with the highest $D$-efficiency is then selected.

Recall that the $D$-optimal design measure over an $m$-ball puts a measure $2 /\{(m+1)$ $(m+2)\}$ at the origin; that is, the center point and the rest of the design weight is uniformly spread over the $m$-ball of unit radius that forms the boundary of the experimental region (see, e.g., Kiefer 1960). The determinant of the information matrix, say $M_{0}$, under this $D$-optimal design measure is then given by

$$
\operatorname{det}\left(M_{0}\right)=\frac{2^{m}(m+3)^{\frac{1}{2} m(m+3)}}{(m+1)^{\frac{1}{2}(m+1)(m+2)}(m+2)^{m(m+2)}} .
$$

With reference to the design $d, l$ et $X_{d}$ denote the $n \times p$ design matrix of $d$, where, as before, the ith row of $X_{d}$ is written as

$$
\left(1, x_{i 1}^{2}, x_{i 2}^{2}, \ldots, x_{i m}^{2}, x_{i 1}, x_{i 2}, \ldots, x_{i m}, x_{i 1} x_{i 2}, \ldots, x_{i, m-1} x_{i m}\right)
$$

and $p=(m+2)(m+1) / 2$ is the number of parameters in the full second-order model. Define $M_{d}=n^{-1} X_{d}^{\prime} X_{d}$. Since the experimental region is an $m$-ball of radius unity, the levels of the design $d$ have to be suitably scaled so that all points are either inside the $m$-ball of radius unity or at its boundary.

The percent $D$-efficiency of the design $d$ relative to an approximate $D$-optimal design is then given by 


$$
\% D-\text { efficiency }=100\left(\frac{\operatorname{det}\left(M_{d}\right)}{\operatorname{det}\left(M_{0}\right)}\right)^{1 / p},
$$

where $\operatorname{det}\left(M_{0}\right)$ is as in Eq. (2) and $\operatorname{det}\left(M_{d}\right)$ is the determinant of $M_{d}$. The expression for the $D$-efficiency given by Eq. (3) in fact provides a conservative lower bound to the actual $D$-efficiency of the design $d$, as this is computed relative to a hypothetical optimal design that is unattainable with a finite number of observations.

For each of the new designs, we also compute the values of $Q^{*}$, a measure of second-order rotatability, proposed by Draper and Pukelsheim (1990). For completeness, this measure is briefly explained in the following. Consider an $N$-point second-order rotatable design involving $m$ factors with second-order moments $\lambda_{2}=N^{-1} \Sigma_{u=1}^{N} x_{i u}^{2}$ and $\lambda_{4}=N^{-1} \Sigma_{u=1}^{N} x_{i u}^{2} x_{j u}^{2}$, where $x_{i u}$ is the level of the $i$ th factor in the $u$ th design point, $1 \leq i \leq m, 1 \leq u \leq N$. The moment matrix $V$ (a square matrix of order $\left(m^{2}+m+1\right)$ ) can be written as

$$
V=V_{0}+\lambda_{2}(3 m)^{1 / 2}+\lambda_{4}\{3 m(m+2)\}^{1 / 2} V_{4}
$$

where the (symmetric) matrices $V_{0}, V_{2}$, and $V_{4}$ are defined as follows: $V_{0}$ consists of an entry 1 in the $(1,1)$ th position and zeros elsewhere, $V_{2}$ consists of an entry $(3 m)^{-1 / 2}$ in each of the $3 m$ positions corresponding to the second-order moments in $V$ and zeros elsewhere, and $V_{4}$ consists of $3\{3 m(m+2)\}^{-1 / 2}$ in the $m$ positions corresponding to pure fourth-order moments, with $\{3 m(m+2)\}^{-1 / 2}$ in the $3 m(m-1)$ positions corresponding to mixed even fourth-order moments in $V$ and zeros elsewhere. Now consider an arbitrary $N$-point secondorder design with moment matrix $A$ and define $\bar{A}=V_{0}+\operatorname{tr}\left(A V_{2}\right) V_{2}+\operatorname{tr}\left(A V_{4}\right) V_{4}$, where $\operatorname{tr}(\cdot)$ stands for the trace of a square matrix. The rotatablity measure $Q^{*}$ is then given by

$$
Q^{*}=\left\{\operatorname{tr}\left(\bar{A}-V_{0}\right)^{2}\right\} /\left\{\operatorname{tr}\left(A-V_{0}\right)^{2}\right\} .
$$

For any second-order design, $Q^{*} \leq 1$, with equality if and only if the design is second-order rotatable.

\section{Results and Discussion}

Table 1 displays 24 orthogonally blocked three-level second order designs for 5-11 factors with 100 runs or less. This table includes, for each design, the number of factors $m$, the number of parameters $p$ in the second-order response surface model, the design identification, the associated $t$-resolvable RGD with parameters $(v, k, r)$, the total number of runs $n$, the number of center runs $n_{0}$ (which is also the number of orthogonal blocks of each design as a center run is given to each block), the $D$-efficiency, and the rotatability measure $Q^{*}$. To show the relative advantage of the new designs cataloged in Table 1 over the orthogonally blocked BBDs on the design run sizes, the latter are also given in this table. Given next are some general observations for the new designs in Table 1:

1. Although all the designs in Table 1 are available with at most 100 runs, they have reasonably high $D$-efficiencies and rotatability measures in most cases. With the exception of two designs $5 \mathrm{c}$ and $11 \mathrm{~b}$, the $D$-efficiencies of these designs are between 70 and $100 \%$. Note that since the $D$-efficiency is computed relative to a possibly hypothetical optimal 
Table 1

Goodness measures of new designs

\begin{tabular}{|c|c|c|c|c|c|c|c|}
\hline$m$ & $p$ & Design $^{\ddagger}$ & $t$ & $(v, k, r)$ & $n\left(n_{0}^{\dagger}\right)$ & D-eff & $\mathrm{Q}^{*}$ \\
\hline \multirow[t]{6}{*}{5} & \multirow[t]{6}{*}{21} & $5 \mathrm{a}$ & 3 & $(5,3,6)$ & $42(2)$ & 87.93 & 0.9351 \\
\hline & & $5 b$ & 3 & $(5,3,9)$ & $63(3)$ & 94.39 & 0.9802 \\
\hline & & $5 c$ & 2 & $(6,3,4)$ & $34(2)$ & 68.97 & 0.9644 \\
\hline & & $5 d$ & 2 & $(6,3,6)$ & $51(3)$ & 73.88 & 0.9835 \\
\hline & & $5 e$ & 2 & $(6,3,8)$ & $68(4)$ & 75.66 & 0.9908 \\
\hline & & BBD & 2 & $(5,2,4)$ & $42(2)$ & 98.83 & 0.9974 \\
\hline \multirow[t]{4}{*}{6} & \multirow[t]{4}{*}{28} & $6 a$ & 2 & $(6,3,4)$ & $34(2)$ & 75.95 & 0.9240 \\
\hline & & $6 \mathrm{~b}$ & 2 & $(6,3,6)$ & $51(3)$ & 91.49 & 0.9643 \\
\hline & & $6 c$ & 2 & $(6,3,8)$ & $68(4)$ & 96.38 & 0.9858 \\
\hline & & BBD & - & $(6,3,3)$ & $42(2)$ & 94.61 & 0.9905 \\
\hline \multirow[t]{3}{*}{7} & \multirow[t]{3}{*}{36} & $7 \mathrm{a}$ & 3 & $(7,3,6)$ & $58(2)$ & 99.93 & 1.0000 \\
\hline & & $7 b$ & 3 & $(7,3,9)$ & 87 (3) & 97.67 & 0.9854 \\
\hline & & BBD & - & $(7,3,3)$ & $58(2)$ & 99.93 & 1.0000 \\
\hline \multirow[t]{7}{*}{8} & \multirow[t]{7}{*}{45} & $8 \mathrm{a}$ & 3 & $(8,3,6)$ & $66(2)$ & 84.99 & 0.9479 \\
\hline & & $8 b$ & 3 & $(8,3,9)$ & 99 (3) & 95.38 & 0.9827 \\
\hline & & $8 c$ & 3 & $(8,4,6)$ & $98(2)$ & 82.57 & 0.9778 \\
\hline & & $8 d$ & 2 & $(8,4,6)$ & 99 (3) & 83.14 & 0.9778 \\
\hline & & $8 \mathrm{e}$ & 1 & $(9,3,5)$ & $65(5)$ & 69.43 & 0.9581 \\
\hline & & $8 \mathrm{f}$ & 1 & $(9,3,6)$ & $78(6)$ & 72.97 & 0.9770 \\
\hline & & $8 \mathrm{~g}$ & 1 & $(9,3,8)$ & $100(4)$ & 82.81 & 0.9985 \\
\hline \multirow[t]{4}{*}{9} & \multirow[t]{4}{*}{55} & $9 a$ & 1 & $(9,3,5)$ & $65(5)$ & 79.07 & 0.9465 \\
\hline & & $9 b$ & 1 & $(9,3,6)$ & $78(6)$ & 85.35 & 0.9710 \\
\hline & & $9 \mathrm{c}$ & 2 & $(9,3,8)$ & $100(4)$ & 98.18 & 0.9985 \\
\hline & & $\mathrm{BBD}$ & 1 & $(9,3,5)$ & $130(10)$ & 91.86 & 0.9924 \\
\hline \multirow[t]{3}{*}{10} & \multirow[t]{3}{*}{66} & $10 \mathrm{a}$ & 3 & $(10,3,6)$ & $82(2)$ & 80.63 & 0.9466 \\
\hline & & $10 \mathrm{~b}$ & 3 & $(10,5,6)$ & $98(2)$ & 80.81 & 0.9563 \\
\hline & & BBD & - & $(10,4,4)$ & $162(2)$ & 93.46 & 0.9928 \\
\hline \multirow[t]{2}{*}{11} & \multirow[t]{2}{*}{78} & $11 \mathrm{a}$ & 3 & $(11,3,6)$ & $90(2)$ & 70.64 & 0.9334 \\
\hline & & $11 \mathrm{~b}$ & 2 & $(12,3,6)$ & 99 (3) & 67.06 & 0.9393 \\
\hline
\end{tabular}

Number of center points (which is also the number of orthogonal blocks).

Orthogonally blocked BBDs for 8 and 11 factors are not available in Box and Behnken (1960).

design, the efficiencies reported in Table 1 are a lower bound to the actual $D$-efficiency of the design.

2. Designs with parameters as in designs 5a, 5d, 5c, 6b, 6c, 8a, 8b, 9a, 10a, and 11a of Table 1 also appear in Dey and Kole (2013). With the exception of two designs 9a and $8 \mathrm{a}$, the new designs show substantial improvement in $D$-efficiency over the corresponding designs of Dey and Kole. For example, while the $D$-efficiencies of our designs $8 \mathrm{~b}$ (for eight factors in 99 runs and three orthogonal blocks) and 10a (for 10 factors in 82 runs and two orthogonal blocks) are $95.38 \%$ and $80.63 \%$, respectively, the ones of the corresponding Dey-Kole designs are only $82.85 \%$ and $67.35 \%$, respectively. 
3. Designs 7a and 9c were constructed manually from resolvable balanced IBDs with repeated blocks. Similarly, while 7a could be obtained from the algorithm, 9c was constructed manually. These designs are not new: $7 \mathrm{a}$ with a different number of center runs has appeared in Box and Behnken (1960) and Dey and Kole (2013); 9c with a different number of center runs has appeared in Nguyen and Borkowski (2008).

4. Designs 5c, 5d, 5e, 8e, 8f, 8g, and $11 \mathrm{~b}$ were obtained by deleting one column from designs with a larger number of factors. Some new designs in Morris (2000) and Dey (2009) were constructed this way.

5. Some designs in Table 1 use resolvable RGDs with block sizes other than three and fractional factorial other than a half fraction of a $2^{3}$ : Designs $8 \mathrm{c}$ and $8 \mathrm{~d}$ use resolvable RGDs with block size four and a half fraction of a $2^{4}$, and design $10 \mathrm{~b}$ uses a resolvable RGD with block size five and a quarter fraction of a $2^{5}$.

6. With the exception of the five-factor BBD, all BBDs have higher $D$-efficiency and $Q^{*}$ values than the proposed designs. But these require a substantially larger number of runs. The five-factor BBD, however, contains factors with too many zero-level appearances, which might not be attractive to experimenters.

We conclude this section by pointing out some useful online resources on response surface designs. These include http://designcomputing.net/bbd/, http://designcomputing.net/ scd/, and http://iasri.res.in/design/Response\%20Surface/RS_Home.html.

\section{Concluding Remarks}

This article gives a catalog of designs of 24 orthogonally blocked three-level second order designs for 5-11 factors involving 100 runs or less. All designs have the QDF property and reasonably high $D$-efficiencies. Designs with number of factors larger than 11 can be constructed by the method in this article. However, these designs require more than 100 runs, which might not be useful in practice. For experiments with a large number of factors, we recommend the use of the first-order designs in the first stage or screening stage and the designs in this article only in the second stage of the experiment. The runs for all the designs in Table 1 are available at http://designcomputing.net/smallBBD/.

\section{Funding}

The authors thank a referee for making constructive comments on an earlier version. The work of A. Dey was supported by the National Academy of Sciences, India, under the Senior Scientist program of the Academy. The support is gratefully acknowledged.

\section{References}

Box, G. E. P., and D. W. Behnken. 1960. Some new three level designs for the study of quantitative variables. Technometrics, 2, 455-475.

Box, G. E. P., and N. R. Draper. 2007. Response surfaces, mixtures and ridge analyses, 2nd ed. New York, NY: Wiley.

Box, G. E. P., and K. B. Wilson. 1951. On the experimental attainment of optimum conditions. $J . R$. Stat. Soc. Ser. B, 13, 1-45.

Dey, A. 2009. Orthogonally blocked three-level second order designs. J. Stat. Plan. Inference, 139, 398-3705.

Dey, A. 2010. Incomplete block designs. Hackensack, NJ: World Scientific. 
Dey, A., and B. Kole. 2013. Small three-level second-order designs with orthogonal blocks. J. Stat. Theor. Pract., 7, 745-752.

Draper, N. R., and F. Pukelsheim. 1990. Another look at rotatability. Technometrics, 32, 195-202.

John, J. A., and T. J. Mitchell. 1977. Optimal incomplete block designs. J. R. Stat. Soc. Ser. B, 39, $39-43$.

Kiefer, J. 1960. Optimum experimental designs V, with applications to systematic and rotatable designs. Proc. Fourth Berkeley Symp., 1, 381-405.

Morris, M. D. 2000. A class of three-level experimental designs for response surface. Technometrics, $42,111-121$.

Nguyen, N.-K. 1994. Construction of optimal block design by computer. Technometrics, 36, 300-307.

Nguyen, N.-K., and K. L. Blagoeva. 2010. Incomplete block designs. In International Encyclopedia of Statistical Science, ed. L. Miodrag, 653-655. New York, NY: Springer.

Nguyen, N.-K., and J. J. Borkowski. 2008. New 3-level response surface designs constructed from incomplete block designs. J. Stat. Plan. Inference, 138, 294-305.

Nguyen, N.-K., and D. K. J. Lin. 2011. A note on small composite designs for sequential experimentation. J. Stat. Theor. Pract., 5, 109-117. 